\title{
Bubble dynamics atop an oscillating substrate: Interplay of compressibility and contact angle hysteresis
}

Irina S. Fayzrakhmanova, Arthur V. Straube, and Sergey Shklyaev

Citation: Phys. Fluids 23, 102105 (2011); doi: 10.1063/1.3650280

View online: http://dx.doi.org/10.1063/1.3650280

View Table of Contents: http://pof.aip.org/resource/1/PHFLE6/v23/i10

Published by the American Institute of Physics.

\section{Related Articles}

Bubble growth by injection of gas into viscous liquids in cylindrical and conical tubes

Phys. Fluids 23, 102102 (2011)

On the dynamics and breakup of a bubble rising in a turbulent flow

Phys. Fluids 23, 103301 (2011)

Jets in viscous bubbles

Phys. Fluids 23, 091103 (2011)

Preferential concentration and rise velocity reduction of bubbles immersed in a homogeneous and isotropic turbulent flow

Phys. Fluids 23, 093301 (2011)

On the evolution of spherical gas interfaces accelerated by a planar shock wave

Phys. Fluids 23, 084104 (2011)

\section{Additional information on Phys. Fluids}

Journal Homepage: http://pof.aip.org/

Journal Information: http://pof.aip.org/about/about_the_journal

Top downloads: http://pof.aip.org/features/most_downloaded

Information for Authors: http://pof.aip.org/authors

\section{ADVERTISEMENT}

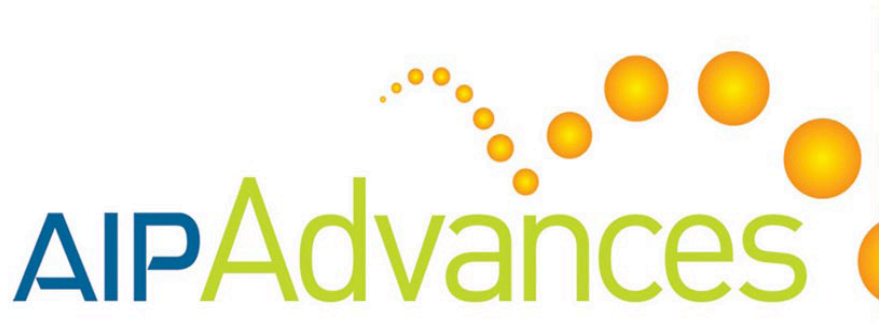

Submit Now

Explore AIP's new

open-access journal

Article-level metrics now available

Join the conversation!

Rate \& comment on articles 


\title{
Bubble dynamics atop an oscillating substrate: Interplay of compressibility and contact angle hysteresis
}

\author{
Irina S. Fayzrakhmanova, ${ }^{1}$ Arthur V. Straube, ${ }^{2,3, a)}$ and Sergey Shklyaev ${ }^{3,4}$ \\ ${ }^{1}$ Department of Mathematics, Technion—Israel Institute of Technology, Haifa 32000, Israel; \\ Department of General Physics, Perm State Technical University, Perm 614990, Russia; and \\ Institute of Continuous Media Mechanics UB RAS, 1 Ac. Korolev St., Perm 614013, Russia \\ ${ }^{2}$ Department of Physics, Humboldt University of Berlin, Newtonstr. 15, Berlin D-12489, Germany \\ ${ }^{3}$ Department of Physics and Astronomy, University of Potsdam, Karl-Liebknecht-Str. 24/25, \\ Potsdam D-14476, Germany \\ ${ }^{4}$ Department of Chemical Engineering, California Institute of Technology, Pasadena, California 91125, USA
}

(Received 13 July 2011; accepted 21 September 2011; published online 19 October 2011)

\begin{abstract}
We consider a sessile hemispherical bubble sitting on the transversally oscillating bottom of a deep liquid layer and focus on the interplay of the compressibility of the bubble and the contact angle hysteresis. In the presence of contact angle hysteresis, the compressible bubble exhibits two kinds of terminal oscillations: either with the stick-slip motion of the contact line or with the completely immobile contact line. For the stick-slip oscillations, we detect a double resonance, when the external frequency is close to eigenfrequencies of both the breathing mode and shape oscillations. For the regimes evolving to terminal oscillations with the fixed contact line, we find an unusual transient resembling modulated oscillations. (c) 2011 American Institute of Physics. [doi:10.1063/1.3650280]
\end{abstract}

\section{INTRODUCTION}

Recent years have shown keen interest in the dynamics of sessile droplets and bubbles on oscillated substrate. ${ }^{1-7}$ While the ability to predict the contact line motion and, therefore, to control the wetting processes is generally important for technological applications, ${ }^{8,9}$ these setups can also be used to manipulate small droplets over surfaces. ${ }^{10-12}$ Instead of substrate vibrations, oscillations of drops and bubbles can be induced by electrowetting, see Refs. 13 and 14 and references therein. Apart from the ability to manipulate sessile objects, these techniques also admit the possibility of mixing. ${ }^{13,15,16}$

Although in many of these applications, the contact line (and/or contact angle) is not static, theoretical models often neglect this dynamic feature or treat it in a simplified way. Independent of how oscillations are induced, the behavior of a sessile droplet or a bubble is significantly determined and often is completely slaved by the dynamics of the triple line. For instance, a simplified model of a compressible bubble atop vibrated substrate that neglects the coupling with the substrate predicts trivial volume oscillations. As the real picture is much more intricate, ${ }^{6}$ this rough but representative example testifies to the necessity of finer models of the contact line motion. The importance of this argument is further augmented, when quantitative agreement between the theory and experiment is required, see also Sec. V.

The purpose of this study is to outline generic features caused by the interaction of such factors as compressibility and contact angle hysteresis, by looking at a relatively simple theoretical model that allows for a deep insight. More precisely, this can be achieved by applying an effective

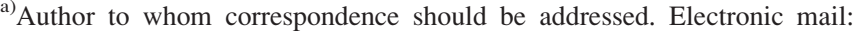
straube@physik.hu-berlin.de.
}

boundary condition suggested by Hocking, ${ }^{17,18}$ which correctly mimics the motion of the contact line and involves specific dissipation caused by this motion. Such an idealized system as a droplet (or a bubble) hemispherical in equilibrium admits a systematic analytical or semianalytical treatment. The latter issue is important for obtaining a deep insight, which is typically lost when one resorts to direct numerical simulations. This strategy has recently been applied to separately explore the impact of the compressibility of bubble $^{6}$ and the contact angle hysteresis. ${ }^{7}$

Apart from shape oscillations typical of any deformable object, a compressible bubble additionally possesses the breathing eigenmode corresponding to radial pulsations. For a sessile bubble, the contact line dynamics causes the interaction of this mode with the eigenmodes of shape oscillations even within the linear problem, ${ }^{6}$ contrary to the necessarily nonlinear coupling for a spherical bubble. ${ }^{19}$ Another prominent feature caused by compressibility is a "double resonance," which means that under certain conditions, two modes simultaneously, one of the modes of shape oscillations and the breathing mode, are resonantly amplified. In this regime, bubble oscillations can be represented as such a superposition of these two modes that the contact line remains almost fixed, which is completely independent of wetting properties.

The issue of contact angle hysteresis has been recently addressed in another study dealing with an incompressible droplet. $^{7}$ As the contact line moves only when the contact angle exceeds a certain critical value, the stick-slip dynamics is observed: the system switches periodically between the phases with the sliding and the fixed contact line. The contact angle hysteresis causes another noteworthy effect. It is known that in the nonhysteretic limit, ${ }^{4}$ no contact line motion exists at a discrete number of "antiresonant" frequencies, which are independent of wetting properties. The 
contact angle hysteresis transforms these discrete frequencies into antiresonant frequency bands of finite width. The dynamics corresponds to stick-slip motion outside the bands and to oscillations with the pinned contact line within the bands. The contact angle hysteresis has been shown to be responsible for a nontrivial time dependence of the contact angle, which is in qualitative agreement with recent experimental observations. ${ }^{1}$

In this study, we report on the interplay of the compressibility and the contact angle hysteresis. Particularly, we show that such coupling results not only in a simple combination of known effects. More importantly, it can also lead to a novel generic feature, which is not possible in previous setups, ${ }^{6,7}$ where these two factors come into play separately. The paper is outlined as follows. In Sec. II, we formulate the problem and in Sec. III, we describe the method of solution. Section IV is devoted to interpretation of results, which is finally followed by conclusions in Sec. V.

\section{PROBLEM STATEMENT}

Consider a gaseous bubble attached to the bottom of a liquid layer of thickness $H$ with a free upper surface. The bubble is assumed hemispherical, with a radius $R$ in equilibrium, implying that the equilibrium contact angle $\chi_{e q}=\pi / 2$. We focus on the case of "deep water," $R \ll H$. The rigid substrate is subject to transverse oscillations with an amplitude $a$ and a frequency $\omega$, see Fig. 1. The amplitude is considered small $\left(a \ll R^{2} / H\right)$ to linearize the governing equations and boundary conditions except for the Hocking condition, see Eq. (1) below. We assume that the frequency $\omega$ is high to neglect the dissipative effects caused by acoustic irradiation, viscous and heat dissipation, ${ }^{20}$ see the corresponding requirements, Eq. (2) in Ref. 6. At the same time, $\omega$ is considered comparable with both the eigenfrequencies of shape and volume oscillations for the bubble. The contact line dynamics is accounted via Hocking's boundary condition, ${ }^{18}$ which includes the contact angle hysteresis.

Following Ref. 6, we use $\sqrt{\rho R^{3} / \sigma}, R, a H / R, \rho a \omega^{2} H$, and $a H \sqrt{\sigma /\left(\rho R^{3}\right)}$ as the scales for the time, length, deviation of bubble surface from its equilibrium position, pressure, and velocity potential, respectively. Here, $\sigma$ is the surface tension and $\rho$ is the liquid density. We work in the spherical coordinates $r, \vartheta$, and $\alpha$ with the origin in the center

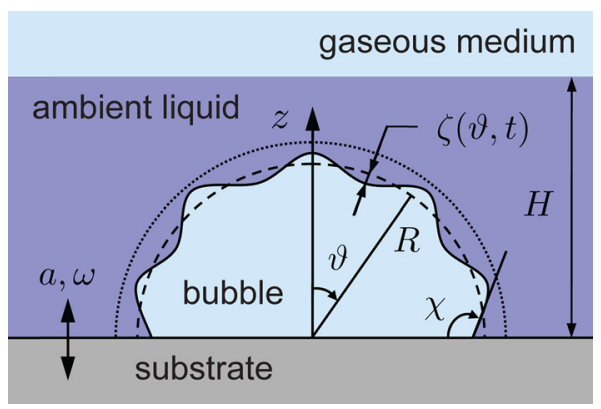

FIG. 1. (Color online) Sketch of a bubble sitting on the transversally oscillating bottom of a deep liquid layer, $H \gg R$. The dashed line shows the equilibrium shape and the dotted line indicates existence of radial (volume) oscillations. of the bubble and restrict our analysis to the axisymmetric problem. In the reference frame of the substrate, the system is described by the equations (cf. Ref. 6)

$$
\begin{aligned}
& p=-\frac{1}{\Omega^{2}} \frac{\partial \varphi}{\partial t}+\cos \Omega t, \quad \nabla^{2} \varphi=0 \\
& \vartheta=\frac{\pi}{2}: \frac{\partial \varphi}{\partial \vartheta}=0 \\
& r=1: \frac{\partial \zeta}{\partial t}=\frac{\partial \varphi}{\partial r}, \quad \Omega^{2} p+\Pi_{0}\langle\zeta\rangle=\left(\nabla_{\vartheta}^{2}+2\right) \zeta, \\
& r=1, \quad \vartheta=\frac{\pi}{2}: \frac{\partial \zeta}{\partial t}=\left\{\begin{array}{l}
\lambda\left(\gamma-\gamma_{c}\right), \gamma>\gamma_{c}, \\
0,|\gamma| \leq \gamma_{c}, \\
\lambda\left(\gamma+\gamma_{c}\right), \gamma<-\gamma_{c},
\end{array}\right. \\
& r \rightarrow \infty: \varphi \rightarrow 0, \\
& \nabla_{\vartheta}^{2}=\frac{1}{\sin \vartheta} \frac{\partial}{\partial \vartheta}\left(\sin \vartheta \frac{\partial}{\partial \vartheta}\right)
\end{aligned}
$$

Here, $\gamma(t) \equiv\left(\chi-\chi_{e q}\right) R^{2} /(a H)=-\left.(\partial \zeta / \partial \vartheta)\right|_{\vartheta=\pi / 2}$ is the rescaled deviation of the contact angle from its equilibrium value and angle brackets abbreviate the averaging over the bubble surface. According to Hocking's hysteretic condition, Eq. (1d), the contact line remains motionless until $|\gamma|$ exceeds a certain critical value $\gamma_{c}$, which is a dimensionless parameter.

In addition to $\gamma_{c}$, the boundary value problem, Eq. (1), is governed by three dimensionless parameters, introduced in the nonhysteretic study: ${ }^{6}$ the external frequency $\Omega=\sqrt{\rho \omega^{2} R^{3} / \sigma}$, the Hocking (or wetting) parameter $\lambda=\Lambda \sqrt{\rho R / \sigma}$, and the pressure in the bubble $\Pi_{0}=3 n_{p} P_{g} R / \sigma$. Here, $n_{p}$ is the polytropic (e.g., adiabatic) exponent and $P_{g}$ and $\Lambda$ are respectively dimensional pressure in the bubble and the Hocking parameter. As the relative depth of the layer $H / R$ is large, the boundary conditions at the free surface have been replaced by the requirement of the decay of oscillations away from the bubble, Eq. (1e), see discussion in Ref. 6.

\section{METHOD OF SOLUTION}

Although the hysteretic boundary condition at the contact line, Eq. (1d), makes the boundary value problem, Eq. (1), nonlinear, series representations for $\varphi, p$, and $\zeta$ applied earlier [cf. Eq. (21) in Ref. 6] can be used:

$$
\begin{gathered}
\zeta=\sum_{n=0}^{\infty} C_{n}(t) P_{2 n}(\theta), \quad \varphi=-\sum_{n=0}^{\infty} \frac{\dot{C}_{n}(t) P_{2 n}(\theta)}{(2 n+1) r^{2 n+1}} \\
p=\frac{1}{\Omega^{2}} \sum_{n=0}^{\infty} \frac{\ddot{C}_{n}(t) P_{2 n}(\theta)}{(2 n+1) r^{2 n+1}}+\cos \Omega t, \quad \theta \equiv \cos \vartheta .
\end{gathered}
$$

Hereafter, dots denote the time derivatives.

Another representation of $\zeta$ follows from the normal stress balance condition, the second relation in Eq. (1c)

$$
\zeta=\gamma \theta-\sum_{n=1}^{\infty} \frac{\ddot{C}_{n}(t) P_{2 n}(\theta)}{\Omega_{n}^{2}}+\frac{\Omega^{2} \cos \Omega t+\Pi_{0} C_{0}-\ddot{C}_{0}}{2},
$$


where $\Omega_{n}^{2}=(2 n+1)(2 n-1)(2 n+2)$ with $n \geq 1$ are the eigenfrequencies of the shape oscillations of a spherical bubble, which in our study refer to the even modes only. The value $\Omega_{0}^{2}=\Pi_{0}-2$ denotes the eigenfrequency of the volume (breathing) mode for the spherical bubble. It can be readily seen that the first term in Eq. (4) determines the deviation of the contact angle.

The comparison of Eqs. (2) and (4) for $\zeta$ yields a set of ordinary differential equations for $C_{n}$

$$
\begin{aligned}
& \ddot{C}_{0}+\Omega_{0}^{2} C_{0}=-\gamma-\Omega^{2} \cos \Omega t, \\
& \ddot{C}_{n}+\Omega_{n}^{2} C_{n}=\alpha_{n} \Omega_{n}^{2} \gamma(n>0),
\end{aligned}
$$

where $\alpha_{n}$ are introduced as expansion coefficients

$$
\theta=\sum_{n=0}^{\infty} \alpha_{n} P_{2 n}(\theta), \quad \alpha_{n}=-\frac{(4 n+1) P_{2 n}(0)}{(2 n-1)(2 n+2)} .
$$

Equations (5) and (6) should be supplemented with an equation for $\gamma$, which is drawn from Eq. (1d). The convergence of the series for $\zeta$ at $\theta=0$ is very slow. To improve the computational efficiency, we make use of the following idea. We note that the first term in Eq. (6) can be neglected for large $n$. Indeed, this term is of order $\Omega^{2} C_{n}$ (the higher modes of eigenoscillations are not excited) and consequently, it is small in comparison with $\Omega_{n}^{2} C_{n}$. Therefore, starting from a certain $N$, we can put $C_{n} \approx \alpha_{n} \gamma$ and apply Eq. (2)

$$
\frac{\partial \zeta}{\partial t}=\sum_{n=0}^{N} \dot{C}_{n} P_{2 n}(0)+\sum_{n=N+1}^{\infty} \dot{\gamma} \alpha_{n} P_{2 n}(0)=\sum_{n=0}^{N} \dot{\beta}_{n} P_{2 n}(0),
$$

where $\beta_{n}=C_{n}-\gamma \alpha_{n}$ and we used Eq. (7) at $\theta=0$. Relations (8) and (1d) give the desired equation for $\gamma$

$$
\dot{\gamma}=\frac{1}{S_{N}} \sum_{n=0}^{N} \dot{C}_{n} P_{2 n}(0)-\frac{1}{S_{N}}\left\{\begin{array}{l}
\lambda\left(\gamma-\gamma_{c}\right), \gamma>\gamma_{c}, \\
0,|\gamma| \leq \gamma_{c}, \\
\lambda\left(\gamma+\gamma_{c}\right), \gamma<-\gamma_{c},
\end{array}\right.
$$

where $S_{N} \equiv \sum_{n=0}^{N} \alpha_{n} P_{2 n}(0)$. Note that the similar approach can be applied to incompressible droplets equally well and it is even more powerful than that applied earlier in Ref. 7.

The set of Eqs. (5), (6), and (9) was solved numerically with $N=90$. To check the accuracy, we performed tests with $N=100$ and $N=150$, which gave close results with a typical distinction within $2 \%$ between $N=90$ and $N=150$.

\section{RESULTS AND DISCUSSION}

We start our discussion by outlining possible types of dynamics inherent to a compressible bubble with the contact angle hysteresis. Recall that because of dissipative nature of Hocking's condition, Eq. (1d), the system reaches a terminal oscillatory state (a limit cycle) after a certain transient. As has been shown for an incompressible droplet, ${ }^{7}$ the contact angle hysteresis leads to the existence of antiresonant bands. Depending on the parameters $\Omega$ and $\gamma_{c}$ but being completely independent of the wetting parameter, $\lambda$, two characteristic types of terminal oscillations are possible: (1) with the stickslip motion of the contact line and (2) with the contact line

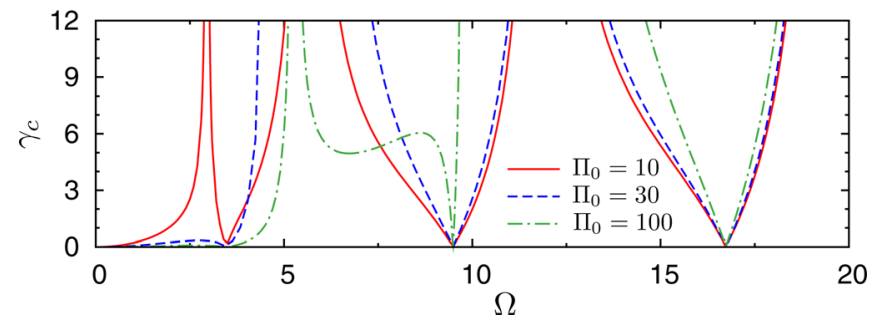

FIG. 2. (Color online) Diagram showing the border $\gamma_{c}(\Omega)$ between the domains of oscillations with the fixed contact line (antiresonant bands, above the curves) and with the stick-slip motion (below the curves), plotted for different $\Pi_{0}$.

pinned, respectively outside and within the antiresonant bands. The border separating the corresponding domains on the plane $\left(\Omega, \gamma_{c}\right)$ is drawn from the partial problem with the pinned contact line. For a compressible bubble, the situation is similar with a difference that now a dependence on the parameter $\Pi_{0}$ appears, see Fig. 2. Note that variation of $\Pi_{0}$ significantly affects the widths of the antiresonant bands.

Before we proceed to the analysis of terminal oscillations, we emphasize an outstanding feature, which concerns transients related to the domains with the fixed contact line (antiresonant bands) in Fig. 2 and is a result of interplay between the compressibility and the contact angle hysteresis. The bubble response strongly resembles modulated oscillations, see Fig. 3. Note that most of the time $|\gamma|<\gamma_{c}$, implying that the contact line is fixed and no damping occurs. The oscillations are subject to damping during the relatively short spells of sliding motion, $|\gamma(t)|>\gamma_{c}$. As a result, in a few hundreds of periods, a specific regime of oscillations in which the amplitude of "modulated" oscillations equals $\gamma_{c}$ is reached; the contact line stops sliding and the oscillations no longer decay.

A closer inspection of the signal $\gamma(t)$ shows that it presents a superposition of the externally imposed oscillation and an eigenmode: $a_{\gamma} \cos \Omega t+\left(\gamma_{c}-a_{\gamma}\right) \cos \omega_{n} t$. Here, $a_{\gamma}=a_{\gamma}\left(\Omega, \Pi_{0}\right)$ and $\omega_{n}=\omega_{n}\left(\Pi_{0}\right)$ are, respectively, the amplitude of forced oscillations of $\gamma(t)$ and the eigenfrequency of oscillations with the fixed contact line. In our example, $a_{\gamma}=1.78, n=1$, and $\omega_{1}=4.05$. Further calculations demonstrate that for any initial conditions that admit the stick-slip motion of the contact line during the transient, only one of eigenmodes is selected - the one with the eigenfrequency $\omega_{n}$ closest to $\Omega$; other eigenmodes decay faster and are eventually "filtered out" due to the Hocking dissipation.

At a first glance, this effect is unexpected, as the decay rates caused by the contact line dynamics are approximately

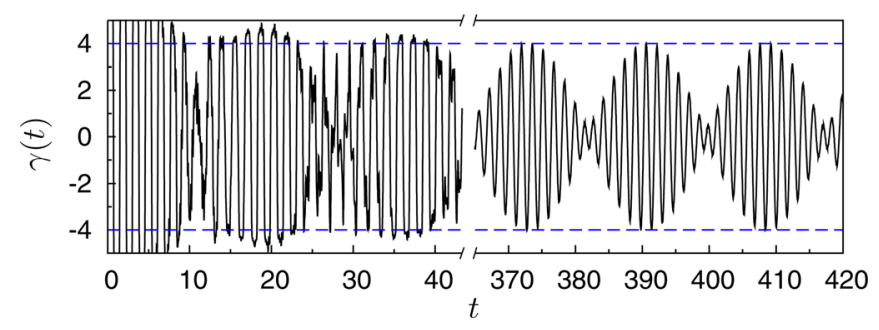

FIG. 3. (Color online) Evolution of $\gamma(t)$ plotted for $\lambda=5, \Pi_{0}=20, \gamma_{c}=4$, and $\Omega=3$.7. 
the same for all the eigenmodes. ${ }^{4,6}$ Therefore, it is not clear why a single eigenmode (and which one exactly) is selected. The only straightforward case in which dissipation becomes vanishingly small for any $\lambda$ is the case of a double resonance, when $\Omega_{n} \approx \Omega_{0}$ and $\Omega$ is close to both these frequencies. ${ }^{6}$ From this perspective, the "leading" eigenmode should be that with the eigenfrequency $\omega_{n}$ closest to the double resonance. However, our calculations show that modulated oscillations exist even away from the double resonance and hence the argumentation should be modified.

What does explain the minimization of dissipation for the leading eigenmode is a consideration of the spells during which the contact line slides. Note first that for any superposition of the forced oscillation and an eigenmode with an eigenfrequency $\omega_{n}$, the evolution of the contact angle can be represented as amplitude-modulated oscillations with the pumping frequency $\Omega$ and the frequency of modulation $\left|\omega_{n}-\Omega\right|$. Then, the dissipation takes place only when the contact line keeps sliding, or, in other words, during the spells with $|\gamma(t)|>\gamma_{c}$. Thus, as becomes clear from Fig. 3, smaller dissipation corresponds to smaller frequencies of modulation, $\left|\omega_{n}-\Omega\right|$, which explains the dominance of the eigenmode with smallest $\left|\omega_{n}-\Omega\right|$ and hence, the origin of modulated oscillations. We stress that this effect is inherent to a compressible bubble only and is not found for a drop. ${ }^{7}$ For an incompressible droplet, the signal $\gamma(t)$ is more complicated, including always several eigenmodes shifted in phases.

Of course, in a real system, other dissipative mechanisms neglected in our study (say, for simplicity, viscosity, with a characteristic time scale $\tau_{\nu} \sim R^{2} / \nu$, where $\nu$ is the kinematic viscosity of the liquid) eventually suppress the eigenmode oscillations. After that, the system approaches the terminal state- the studied earlier linear oscillations with the pinned contact line. ${ }^{6}$ However, such a nontrivial transient is expected to be visible on times smaller than $\tau_{\nu}$. For instance, for a bubble of radius $0.1 \mathrm{~cm}$ in water $\tau_{\nu} \approx 1 \mathrm{~s}$ and the capillary time scale $\tau_{c}=\sqrt{\rho R^{3} / \sigma} \simeq 0.003 \mathrm{~s}$, which means that several hundreds of oscillations take place before the extinction of the leading eigenmode. Beyond the antiresonant bands, the system approaches the terminal state due to the Hocking mechanism of dissipation. Note that the characteristics of the terminal state are only slightly affected by viscosity. The magnitude of such a change can be estimated as $\tau_{c} / \tau_{\nu}$, which provides as small as $\tau_{c} / \tau_{\nu} \simeq 0.003$ for the example above.
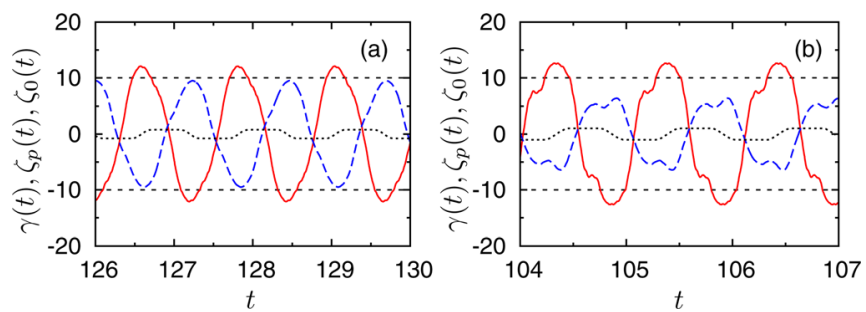

FIG. 4. (Color online) Evolutions of the contact angle, $\gamma(t)$, surface deviations at the pole, $\zeta_{p}(t)$, and on the substrate, $\zeta_{0}(t)$, denoted by solid, dashed, and dotted lines, respectively. The horizontal dashed lines are for $\gamma= \pm \gamma_{c}$. Parameters are $\lambda=5, \Pi_{0}=10$ and $\gamma_{c}=10$, and (a) $\Omega=5.1$ and (b) $\Omega=6.0$.

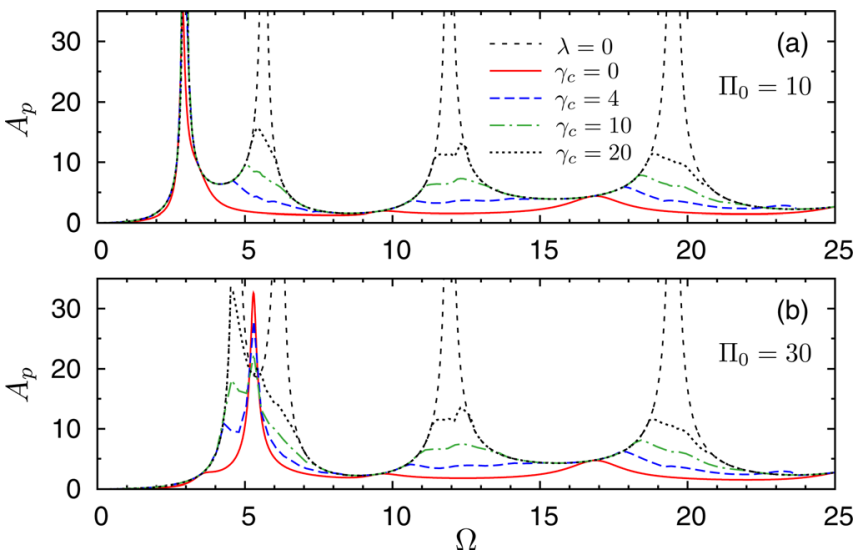

FIG. 5. (Color online) Amplitudes of surface deviation at the pole, $A_{p}$, as functions of frequency $\Omega$ for $\lambda=5$ and different values of $\gamma_{c}$, plotted for $\Pi_{0}=10$ (a) and $\Pi=30$ (b).

We next consider the terminal oscillations with the stick-slip motion at the contact line, which are qualitatively similar to those found for a droplet. ${ }^{7}$ Namely, away from the resonant frequencies, the response is simple. However, even in this case, the oscillations are nonlinear: as ensured by the nonlinearity due to Hocking's condition, generation of higher harmonics occurs. When $\Omega$ is close to $\omega_{n}$, the evolution can become more intricate showing appearance of several local maxima in $\gamma(t)$ and $\zeta_{p}(t) \equiv \zeta(\vartheta=0, t)$ during a period, cf. panels (a) and (b) in Fig. 4. The oscillations of the bubble volume $C_{0}(t)$ remain almost harmonic irrespective of $\lambda$ and $\Omega$.

Despite qualitative similarity with the droplet, ${ }^{7}$ a feature inherent to the bubble oscillations appears, see amplitude response for different values of $\gamma_{c}$ and $\Pi_{0}$ in Fig. 5. Although all the curves for finite $\gamma_{c}$ are between those for the nonhysteretic case, $\gamma_{c}=0$, and the case of $\gamma_{c} \rightarrow \infty$ (or, equivalently, the pinned-end limit, $\lambda=0$ ) as in Ref. 7, we now have the additional parameter $\Pi_{0}$. This parameter can be used to tune $\Omega_{0}$ such that it is close to an eigenfrequency of the shape oscillations, $\Omega_{n}$, when the dissipation drops down. ${ }^{6}$ As a result, at frequencies $\Omega \approx \Omega_{0} \approx \Omega_{n}$, we can expect a double resonance. ${ }^{6}$ The first exact resonance occurs at $\Pi_{0}=14$, when $\Omega_{0}=\Omega_{1}=\sqrt{12}$. As can be seen in Fig. 5, the double resonance is well pronounced for $\Pi_{0}=10$ but no longer present at $\Pi_{0}=30$. Note that the double resonance has two distinctive features: (1) in contrast to usual resonances, the response characteristics of the double resonance are almost independent of $\gamma_{c}$ and (2) compared for finite values of $\gamma_{c}$, its amplitude $\propto\left(\Omega_{0}-\Omega_{n}\right)^{-2}$ is much larger than those for usual resonances.

\section{CONCLUSIONS}

Concluding, we have considered a hemispherical bubble sitting on the transversally oscillating bottom of a deep liquid layer and studied the interplay of the compressibility of the bubble and the contact angle hysteresis. At the heart of our approach is Hocking's hysteretic condition at the triple line. On one hand, it allows us to capture principal features of the contact line dynamics. On the other one, this boundary condition keeps the model relatively simple, which admits 
the possibility of a systematic analysis. We have shown that the combined action of the compressibility and contact angle hysteresis does not simply inherit known effects caused by one of these factors [see points (1) and (2) below] ${ }^{6,7}$ but also leads to a qualitatively novel feature not possible in the previously considered systems [point (3) below].

(1) Similar to an incompressible droplet in the presence of contact angle hysteresis, ${ }^{7}$ the compressible bubble exhibits two kinds of terminal oscillations: either with the stickslip motion of the contact line or with the thoroughly motionless contact line. For both systems, the border separating these regimes of terminal oscillations is independent of the wetting parameter, $\lambda$, and depends on the threshold value of the contact angle $\gamma_{c}$ and on the external frequency $\Omega$. For an incompressible droplet, the oscillations with the pinned contact line and those with the stick-slip motion represent fixed domains on the plane $\left(\Omega, \gamma_{c}\right)$. For a compressible bubble, an additional dependence on the pressure in the bubble, $\Pi_{0}$, appears and this diagram is significantly affected by the value of $\Pi_{0}$, see Fig. 2 .

(2) For the regime of terminal oscillations with the stick-slip motion of the contact line, we end up with the following conclusion. Similar to a compressible bubble with no contact angle hysteresis, ${ }^{6}$ the studied system preserves the effect of double resonance. This happens when the external frequency $\Omega$ is close simultaneously to the frequency of the breathing mode and to one of eigenfrequencies of the shape oscillations (both for a spherical bubble of the same radius), $\Omega_{0} \approx \Omega_{n}$. Interestingly, the oscillations are found to be almost independent of the threshold value of the contact angle, $\gamma_{c}$. We also note that the double resonance is much stronger than usual resonances. From the experimental point of view, the double resonance can be detected by adjusting $\Pi_{0}$ such that $\Omega_{0}\left(\Pi_{0}\right) \approx \Omega_{n}$. The condition $\Omega \approx \Omega_{n}$ is ensured by tuning the external frequency $\Omega$.

(3) For the regime of terminal oscillations with the fixed contact line, we found out an unusual transient resembling modulated oscillations, as in Fig. 3. Any initial conditions that admit the stick-slip motion of the contact line during the transient lead to oscillations presenting a superposition of the external signal with the frequency $\Omega$ and a single eigenmode with the eigenfrequency $\omega_{n}$ closest to $\Omega$. Here, $\omega_{n}$ is the eigenfrequency of the shape oscillations for the hemispherical bubble with the pinned contact line. Although in a real system this eigenmode will ultimately be suppressed (e.g., by viscosity), such a nontrivial transient is expected to be experimentally testable: for instance, for a bubble of radius $0.1 \mathrm{~cm}$ in water several hundreds of oscillations take place before the amplitude modulation is completely washed out. Note that the effect of modulation is possible neither for a bubble without contact angle hysteresis ${ }^{6}$ nor for an incompressible droplet. ${ }^{7}$

More generally, we believe that the potential of Hocking's dynamic boundary condition at the triple line goes beyond the studied problem, including studies in Refs. 4, 6, and 7 , and can be helpful in a different context. We briefly mention a relation to the problem of bubble oscillations generated by means of electrowetting. ${ }^{14}$ Despite the different way of exciting oscillations, the two problems are similar in several aspects.
First, a simple model developed in Ref. 13 for a drop and later modified for a bubble ${ }^{14}$ neglects the coupling to the substrate and, therefore, involves no interaction of different "Rayleigh's" modes (associated with different Legendre polynomials). The interaction of these modes through the substrate is known to shift the eigenfrequencies from the values $\Omega_{n}$, see, e.g., Refs. 4 and 6. Note also that for any finite value of the wetting parameter, $\lambda$, the resonant frequencies do not coincide with the eigenfrequencies. Most likely, this is a reason of a quantitative discrepancy between the experimental measured and theoretical resonant frequencies, which can be fixed via the methodology that we applied in the present study. A comparison of resonant frequencies for different values of $\lambda$ with those measured experimentally would make possible to estimate the value of $\lambda$ for a given experimental system.

Second, the account of viscosity in the theoretical model applied in Ref. 13 can be improved. The contribution into dissipation of the viscous boundary layer developing near the substrate is known (see, e.g., Ref. 21) to prevail over the volume dissipation taken into account in this study. ${ }^{13}$ For this reason, the decay rates should be estimated as being proportional to $\delta=\sqrt{\nu / \omega R^{2}}$ rather than $\delta^{2}$. An example of estimates for a hemispherical drop can be found in Ref. 4. This circumstance may be a reason behind the theoretically overestimated resonant amplitudes. ${ }^{13}$

Finally, we point out that the correct description of the interaction with the substrate can also be an important prerequisite for obtaining the proper picture of the bulk dynamics, for instance, in oscillating bubbly liquids under confinement, see Ref. 22 and references therein. Another example concerns acoustic or electrowetting-driven oscillations of a sessile bubble used to generate streaming flow in the bulk, which presents a way to manipulate small-sized objects indirectly, including capture, propulsion, and release. ${ }^{23-25}$

\section{ACKNOWLEDGMENTS}

The authors gratefully acknowledge support by German Science Foundation (DFG Project No. 436 RUS113/977/01) and Russian Foundation for Basic Research (Project No. 08-01-91959). A.S. additionally acknowledges support by DFG SPP 1164 "Nano- and microfluidics" (DFG Project No. STR 1021/1-2).

${ }^{1}$ X. Noblin, A. Buguin, and F. Brochard-Wyart, "Vibrated sessile drops: Transition between pinned and mobile contact line oscillations," Eur. Phys. J. E 14, 395 (2004).

${ }^{2}$ S. Daniel, M. K. Chaudhury, and P. G. de Gennes, "Vibration-actuated drop motion on surfaces for batch microfluidic processes," Langmuir 21, 4240 (2005).

${ }^{3}$ A. Buguin, F. Brochard, and P.-G. de Gennes, "Motions induced by asymmetric vibrations. The solid/solid case,” Eur. Phys. J. E 19, 31 (2006).

${ }^{4}$ D. V. Lyubimov, T. P. Lyubimova, and S. V. Shklyaev, "Behavior of a drop on an oscillating solid plate,” Phys. Fluids 18, 012101 (2006).

${ }^{5}$ B. Vukasinovic, M. K. Smith, and A. Glezer, "Dynamics of a sessile drop in forced vibration,” J. Fluid Mech. 587, 395 (2007).

${ }^{6}$ S. Shklyaev and A. V. Straube, "Linear oscillations of a hemispherical bubble on a solid substrate," Phys. Fluids 20, 052102 (2008).

${ }^{7}$ I. S. Fayzrakhmanova and A. V. Straube, "Stick-slip dynamics of an oscillated sessile drop," Phys. Fluids 21, 072104 (2009).

${ }^{8}$ P.-G. De Gennes, "Wetting: statics and dynamics," Rev. Mod. Phys. 57, 827 (1985). 
${ }^{9}$ L. Leger and J. F. Joanny, “Liquid spreading," Rep. Prog. Phys. 55, 431 (1992).

${ }^{10}$ T. M. Squires and S. R. Quake, "Microfluidics: Fluid physics at the nanoliter scale," Rev. Mod. Phys. 77, 977 (2005).

${ }^{11}$ X. Noblin, R. Kofman, and F. Celestini, "Ratchetlike motion of a shaken drop," Phys. Rev. Lett. 102, 194504 (2009).

${ }^{12}$ K. John and U. Thiele, "Self-ratcheting Stokes drops driven by oblique vibrations," Phys. Rev. Lett. 104, 107801 (2010).

${ }^{13}$ J. M. Oh, S. H. Ko, and K. H. Kang, "Shape oscillation of a drop in ac electrowetting," Langmuir 24, 8379 (2008).

${ }^{14}$ S. H. Ko, S. J. Lee, and K. H. Kang, "A synthetic jet produced by electrowetting-driven bubble oscillations in aqueous solution," Appl. Phys. Lett. 94, 194102 (2009).

${ }^{15}$ F. Mugele, J.-C. Baret, and D. Steinhauser, "Microfluidic mixing through electrowetting-induced droplet oscillations," Appl. Phys. Lett. 88, 204106 (2006).

${ }^{16}$ R. Shilton, M. K. Tan, L. Y. Yeo, and J. R. Friend, "Particle concentration and mixing in microdrops driven by focused surface acoustic waves," J. Appl. Phys. 104, 014910 (2008).
${ }^{17}$ L. M. Hocking, "The damping of capillary-gravity waves at a rigid boundary," J. Fluid Mech. 179, 253 (1987).

${ }^{18}$ L. M. Hocking, "Waves produced by a vertically oscillating plate," J. Fluid Mech. 179, 267 (1987).

${ }^{19}$ Z. C. Feng and L. G. Leal, "Nonlinear bubble dynamics," Annu. Rev. Fluid Mech. 29, 201 (1997).

${ }^{20} \mathrm{~L}$. van Wijngaarden, "One-dimensional flow of liquids containing small gas bubbles,” Annu. Rev. Fluid Mech. 4, 369 (1972).

${ }^{21}$ C. C. Mei and L. F. Liu, "The damping of surface gravity waves in a bounded liquid," J. Fluid Mech. 59, 239 (1973).

${ }^{22}$ A. V. Straube, "Small-scale particle advection, manipulation, and mixing: Beyond the hydrodynamic scale," J. Phys. Condens. Matter 23, 184122 (2011).

${ }^{23} \mathrm{P}$. Marmottant and S. Hilgenfeldt, "Controlled vesicle deformation and lysis by single oscillating bubbles," Nature (London) 423, 153 (2003).

${ }^{24} \mathrm{~S}$. K. Chung and S. K. Cho, "On-chip manipulation of objects using mobile oscillating bubbles," J. Micromech. Microeng. 18, 125024 (2008).

${ }^{25}$ S. K. Chung, K. Rhee, and S. K. Cho, "Bubble actuation by electrowetting-on-dielectric (EWOD) and its applications: A review,” Int. J. Precis. Eng. Manuf. 11, 991 (2010). 